\title{
Effects of Gd and heat treatments on mechanical properties of $\mathrm{Mg}-x \mathrm{Gd}-1 \mathrm{Zn}-0.4 \mathrm{Zr}$ alloys
}

\author{
X. Zheng ${ }^{1}$, J. $\operatorname{Fan}^{1}$, S. $\operatorname{Tan}^{1,2}$, X. Zhang ${ }^{1,2 *}$ \\ ${ }^{1}$ School of Materials Science and Engineering, Nanjing Institute of Technology, Nanjing 211167, P. R. China \\ ${ }^{2}$ Jiangsu Key Laboratory of Advanced Structural Materials and Application Technology, Nanjing 211167, P. R. China
}

\begin{abstract}
Mg- $x$ Gd-1Zn-0.4Zr ( $x=3,6$, and 9 wt.\%, denoted as GZ31K, GZ61K, and GZ91K) ingots underwent solid solution treatment (T4), solid solution and aging treatments (T6). Microstructures and mechanical properties of the alloys were studied. The results showed that with the increase of $\mathrm{Gd}$ content, the $\beta-(\mathrm{Mg}, \mathrm{Zn})_{3} \mathrm{Gd}$-phase at grain boundaries increased for the as-cast alloys. After T4 treatment, the $\beta$-phase was almost dissolved into matrix, and the needle-like precipitated phase was observed. Block precipitates were formed in the GZ61K and GZ91K alloys after T6 treatment. The microhardness and yield strength of the as-cast alloys increased with the increase of Gd as well as T4 and T6 treatments. Among them, GZ91K-T6 exhibits the best comprehensive mechanical properties, which has the highest hardness $(75.9 \mathrm{HV})$, tensile yield strength $(133 \mathrm{MPa})$, ultimate tensile strength $(261 \mathrm{MPa})$, and moderate elongation ( $15.9 \%)$.
\end{abstract}

Ke y words: magnesium alloy, heat treatment, microstructure, mechanical properties

\section{Introduction}

Biomedical magnesium alloys have good biodegradability, biocompatibility, physical and mechanical properties, and hence, they can be used for orthopedic fixation, cardiovascular stents, and the other short-term implant fields [1-2]. They provide temporary support and progressive degradation during service, which eliminate subsequent surgery and reduce the risk of local inflammation after long-term implantation [3-5]. Therefore, magnesium alloys are expected to replace the traditional non-degradable metals as new generation biomedical implanted materials.

In recent years, most of the research has been devoted to the study of corrosion and mechanical properties of magnesium alloys [6-8]. However, there are few studies on how to significantly improve the corrosion and mechanical properties of magnesium alloys. The mechanical properties have an important influence on the life of the magnesium alloy in the human body, especially for the load bearing. If the mechanical properties decay too fast, it will lead to premature failure of the implants [9]. Therefore, it is very important to study the mechanical behavior of biodegradable magnesium alloys. Alloying and heat treatment are the common methods to improve mechanical properties of magnesium alloys. It has been reported that $\mathrm{Zn}, \mathrm{Ca}$, $\mathrm{Sr}, \mathrm{Zr}$, et al. can significantly strengthen biomedical magnesium alloys mainly due to solution strengthening and grain refinement strengthening [10-13]. Solid solution treatment makes the alloying elements dissolve into matrix, and thus leads to solution strengthening [14]. Moreover, the subsequent aging treatment on the solution treated magnesium alloy can produce tiny precipitated phase dispersed in the matrix and grain boundaries, resulting in the strength improvement by dispersive strengthening $[15,16]$.

Previous studies have also shown that Gd element in magnesium alloy can greatly improve its mechanical properties $[17,18]$. It has been reported that Mg-Gd-Zn-Zr series alloys show favorable uniform corrosion mode, good corrosion resistance, and no cytotoxicity [19-21] owing to unique lamellar structures named stacking faults or long period stacking ordered structures, showing good potential in biodegradable implants. In order to better understand the influences of Gd addition and heat treatments on mechanical properties of these alloys, therefore, in this work, solution treatment and aging treatment were carried out on the $\mathrm{Mg}-x \mathrm{Gd}-1 \mathrm{Zn}-0.4 \mathrm{Zr}(x=3,6$, and 9 wt. $\%)$ alloys,

*Corresponding author: e-mail addresses: xbxbzhang2003@163.com, xbzhang@njit.edu.cn 


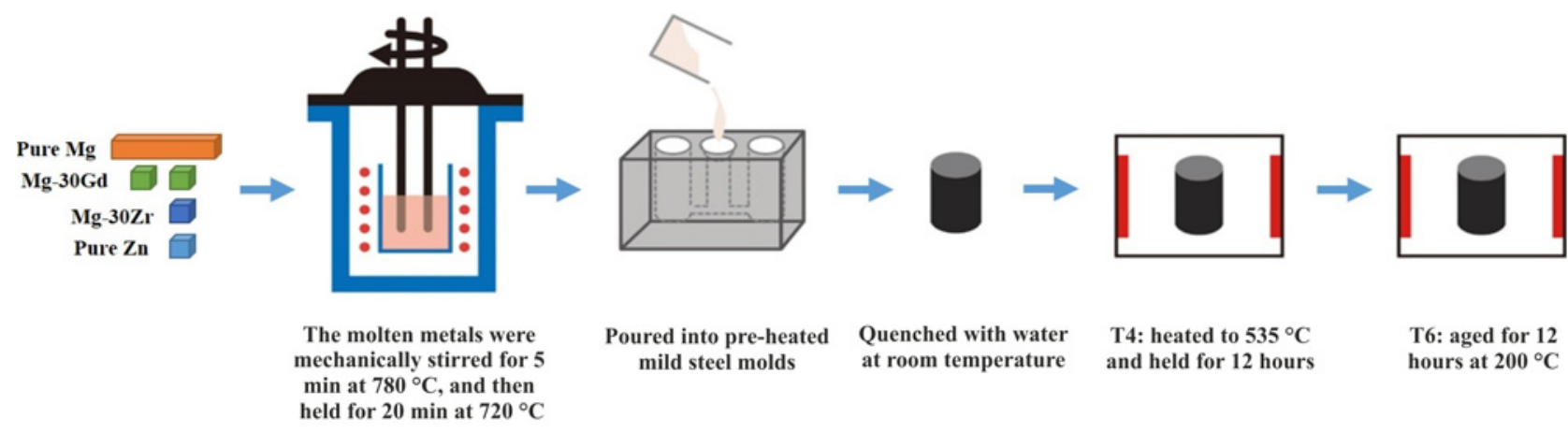

Fig. 1. The preparation processes of the Mg- $x \mathrm{Gd}-1 \mathrm{Zn}-0.4 \mathrm{Zr}$ alloys.

and the microstructure and mechanical properties of the alloys were studied.

\section{Experimental procedure}

The Mg- $x$ Gd-1Zn-0.4Zr $(x=3,6$, and 9 wt. $\%)$ ingots were prepared by conventional casting technique. The preparation processes are shown in Fig. 1. The melting process was performed using an electric resistance furnace under a mixed protective atmosphere, with the successive addition of pure $\mathrm{Mg}$, pure $\mathrm{Zn}$, Mg-30Gd and Mg-30Zr master alloys. The molten metals were mechanically stirred for $5 \mathrm{~min}$ at $780^{\circ} \mathrm{C}$ to obtain uniform melts. After holding for $20 \mathrm{~min}$ at $720^{\circ} \mathrm{C}$, the metals were poured into pre-heated mild steel mold, then quenched with water at room temperature. The as-cast $\mathrm{Mg}-x \mathrm{Gd}-1 \mathrm{Zn}-0.4 \mathrm{Zr}(x=3,6$, and 9 wt.\%) ingots were denoted as GZ31K-F, GZ61K-F, and GZ91K-F, respectively. Solid solution treatment (T4) and solid solution + artificial aging treatments (T6) were performed on the as-cast GZ31K, GZ61K, and GZ91K. The ingots were heated to $535^{\circ} \mathrm{C}$ and held for $12 \mathrm{~h}$ with protective atmosphere, quenched with water to obtain the T4 samples, and then aged for $12 \mathrm{~h}$ at $200^{\circ} \mathrm{C}$ to obtain the T6 samples. The selected heat treatment parameters are based on our previous work [23]. The T4-treated and T6-treated alloys are labeled as GZ31K-T4, GZ61K-T4, GZ91K-T4, GZ31K-T6, GZ61K-T6, and GZ91K-T6, respectively.

The samples were ground by $\mathrm{SiC}$ papers to 1200 grit and polished by $3.5 \mu \mathrm{m}$ diamond paste, then ultrasonically cleaned in anhydrous alcohol and dried in warm air for microstructure observation. After being etched by 4 vol.\% nitric acid in ethanol. The microstructures of different samples were observed using scanning electron microscope (SEM) equipped with energy dispersive X-ray spectroscope (EDS). The phases of the alloys were characterized using X-ray diffraction (XRD) at voltage of $40 \mathrm{kV}$ and a scanning rate of $15^{\circ} \mathrm{min}^{-1}$.

The tensile tests of the alloys were carried out on a materials tensile test machine at ambient temperature with a strain rate of $1.7 \times 10^{-3} \mathrm{~s}^{-1}$, and the tensile tests were triplicated for each condition to show the reproducibility. The tensile sample is in the form of a sheet, with a total length of $54.5 \mathrm{~mm}$. The two ends are square to facilitate machine gripping, the middle part is the effective measuring section, with a gauge width of $3.5 \mathrm{~mm}$, thickness of $2.2 \mathrm{~mm}$, and length of $39.5 \mathrm{~mm}$. The SEM was used to observe the tensile fracture morphologies of the specimens. Microhardness of the alloy was tested by hardness tester under a load of $4.9 \mathrm{~N}$ and duration of $10 \mathrm{~s} .10$ points for each group were conducted to acquire the average microhardness. Moreover, in order to analyze the hardness of different phases, including matrix, $\beta$-phase and precipitated phase, nano hardness was evaluated with a nanoindentor under a maximum normal force of $25 \mathrm{mN}$ and duration of $10 \mathrm{~s}$.

\section{Result and discussion}

\subsection{Microstructure}

Figure 2 shows SEM micrographs of the alloys with different $\mathrm{Gd}$ contents and under different states. As shown in Figs. 2a-c, the microstructures of the as-cast alloys mainly consist of dark $\alpha$-Mg matrix, discontinuous bright $\beta$-phase distributed at the grain boundaries, and lamellar grey structures at the outer edge of matrix grains. According to our previous studies, the lamellar structures in GZ31K-F are stacking faults (SFs) [22], and those in GZ61K-F are long period stacking ordered (LPSO) structures [23]. The LPSO structure is easily formed in the $\mathrm{Mg}-\mathrm{Gd}-\mathrm{Zn}(-\mathrm{Zr})$ alloys with higher Gd addition [24, 25], so the lamellar structure in GZ91K-F is probably LPSO structure. The volume fraction of the $\beta$-phase increases, and the grain size decreases with increasing Gd addition, indicating that Gd plays a positive role in grain refinement.

After T4 treatment, the $\beta$-phase is almost dissolved into $\alpha-\mathrm{Mg}$, needle-like and spherical bright precipitated phases are observed in the grain interiors and 

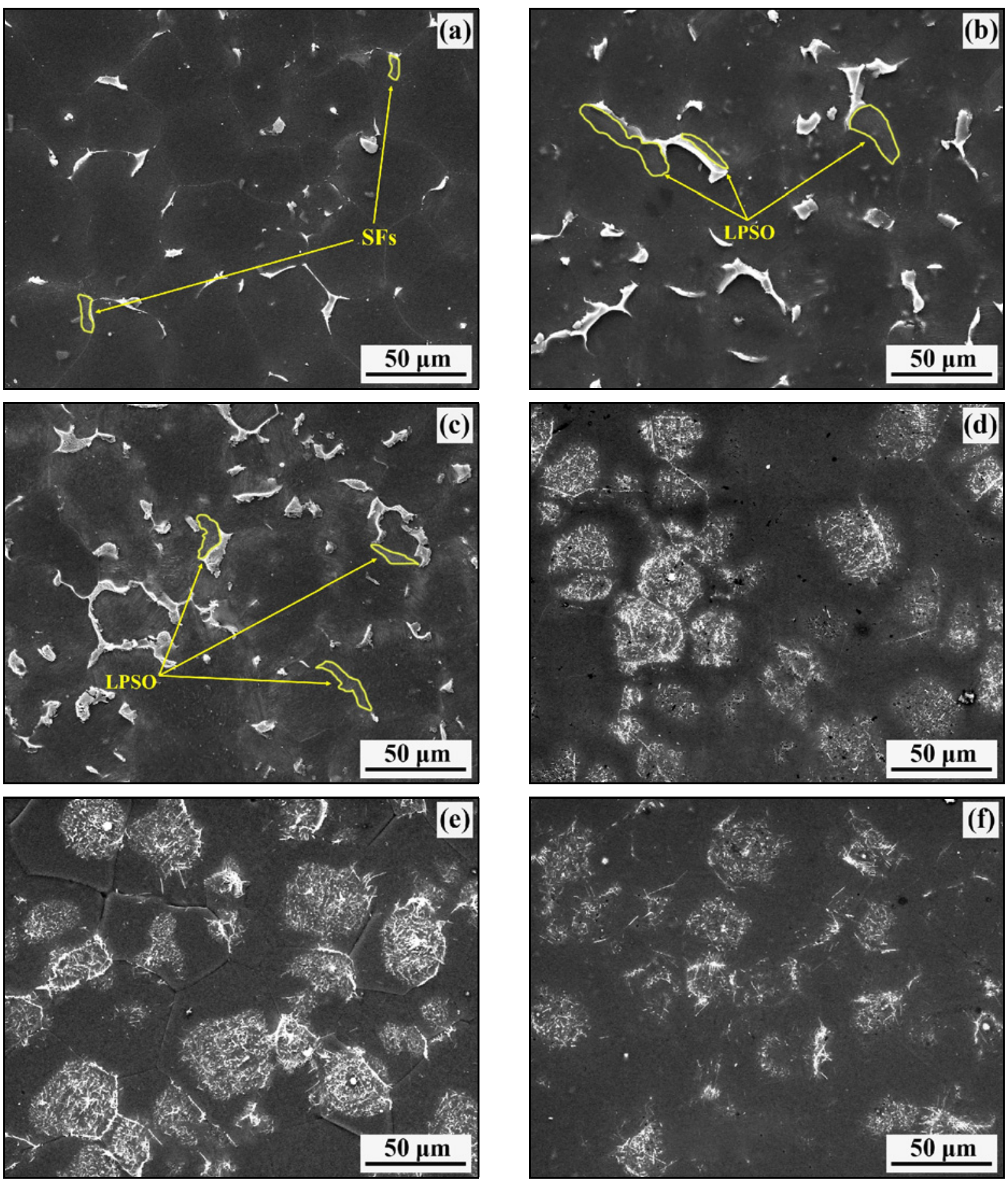

Fig. 2a-f. SEM images of the alloys at different conditions: (a) GZ31K-F, (b) GZ61K-F, (c) GZ91K-F, (d) GZ31K-T4, (e) GZ61K-T4, (f) GZ91K-T4.

grain boundaries. The lamellar SFs or LPSO structures found in the as-cast alloys disappear in the T4-treated alloys, and the grain size shows no obvious change after T4 treatment. The different morphologies of the bright precipitated phase are due to the different orientations of the precipitated particles: The spherical particles are observed when the precipitated particles are perpendicular to the observed plane, and needle-like particles are observed when they are par- allel to the observed plane. Consequently, the morphology of the precipitated phase is needle-like. The precipitated phase is Zr-rich phase which can be confirmed in the previous studies on heat-treated Mg-Gd-Zn-Zr [26] and Mg-Nd-Sr-Zr alloys [27]. It also can be seen from the EDS analysis of Fig. 4c that the structure of the needle-shaped precipitated phase in T6 state is rich in $\mathrm{Zr}$. It is clear the $\beta$-phase almost dissolves into the matrix, transforming the ma- 

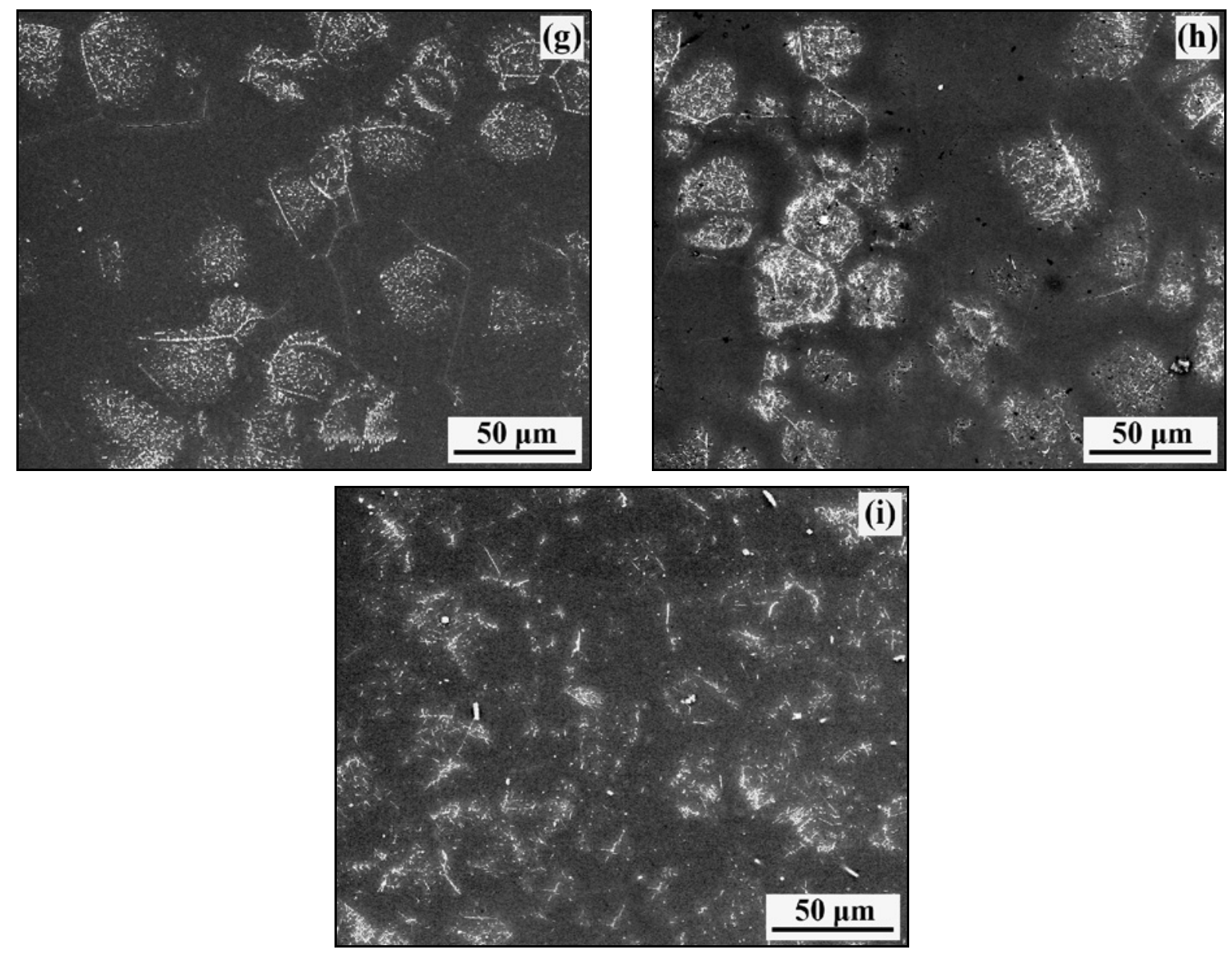

Fig. 2g-i. SEM images of the alloys at different conditions: (g) GZ31K-T6, (h) GZ61K-T6, (i) GZ91K-T6.
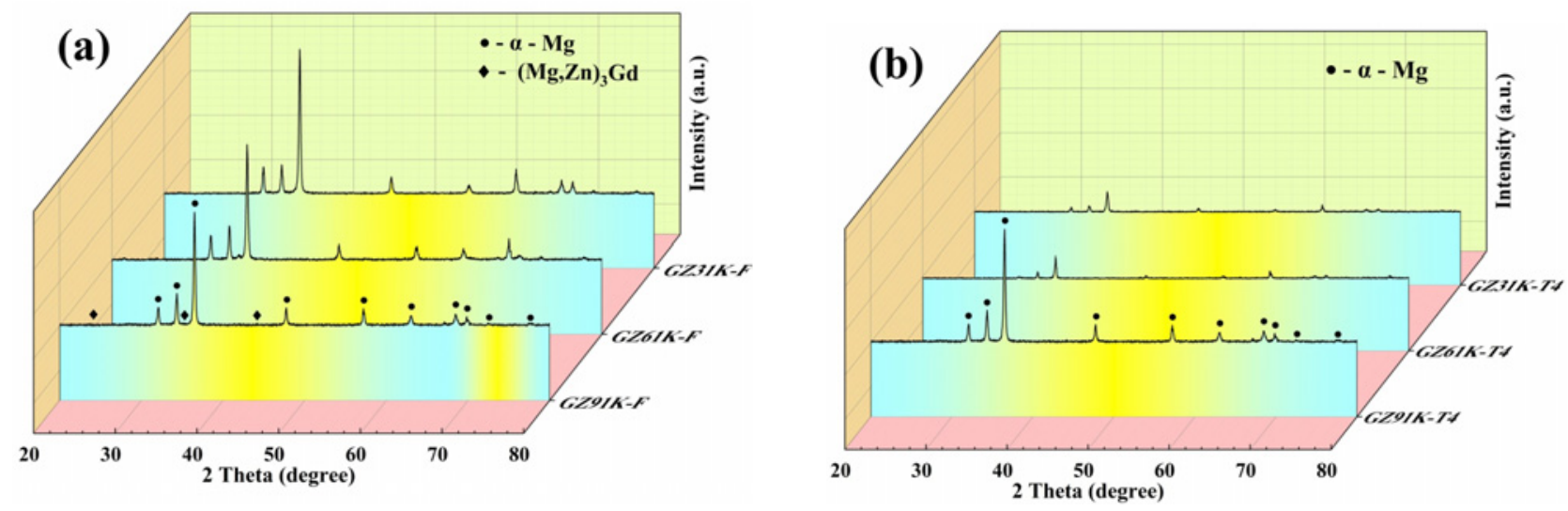

Fig. 3. XRD patterns of the Mg- $x$ Gd-Zn-Zr alloys: (a) as-cast and (b) T4-treated.

trix into supersaturated solid solution after T4 treatment. Because the concentration of solute atoms in SFs or LPSO is much higher than that in the matrix, Gd and Zn solute atoms diffuse from SFs or LPSO to matrix during solution treatment, and thus make the SFs or LPSO structure disappear.

For the T6-treated alloys, the GZ31K shows no apparent microstructural change, but some block par- ticles are observed in the matrix grain interiors and grain boundaries in the GZ61K-T6 and GZ91K-T6 alloys. The volume fraction of bright precipitated phase increases as the Gd addition increases, and it shows higher volume fraction and more homogeneous distribution as compared to those in the T4-treated alloys.

The XRD patterns of the as-cast alloys shown in Fig. 3a indicate that both $\alpha-\mathrm{Mg}$ and $\beta$-(Mg, $\mathrm{Zn})_{3} \mathrm{Gd}-$ 

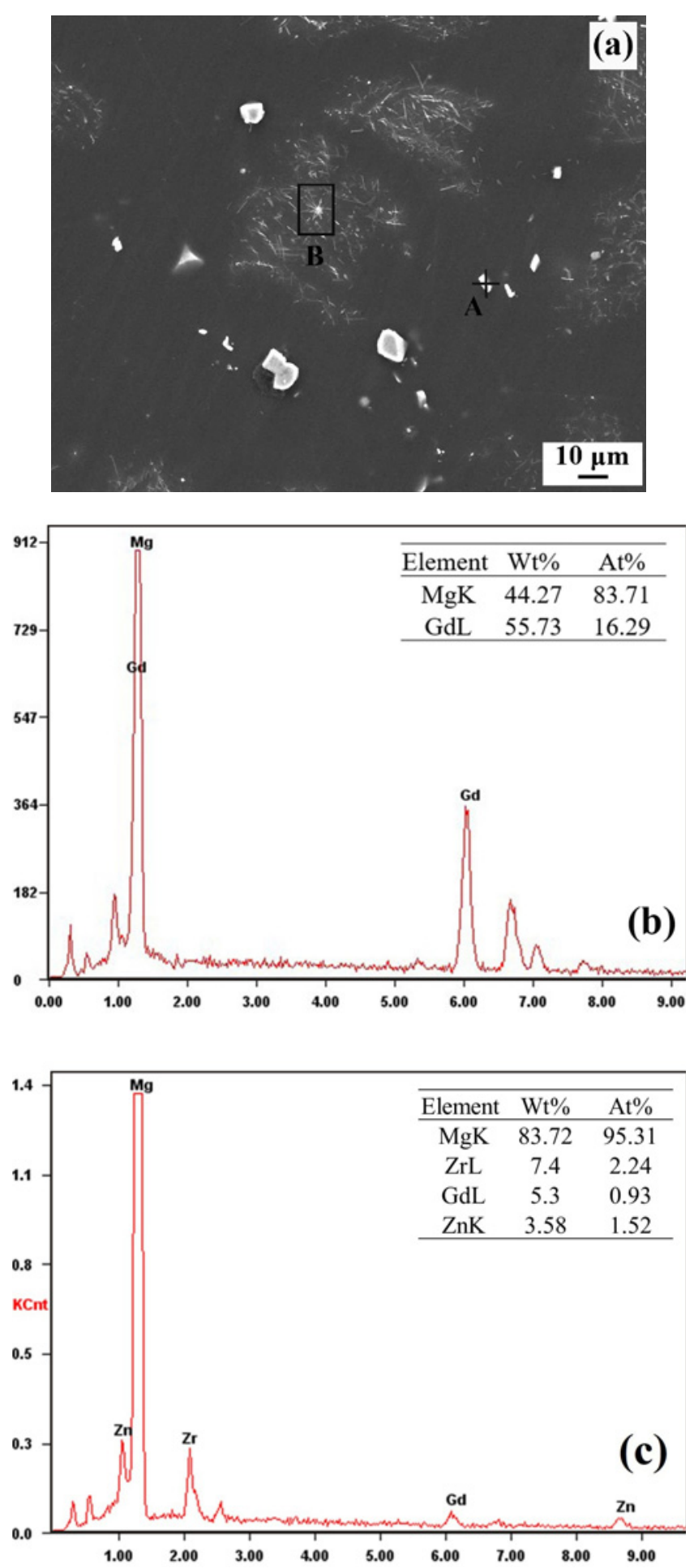

Fig. 4. SEM image of the GZ61K-T6 alloy (a), EDS spectrums of the block phase A (b), and the needle-like phase zone B (c).

-phases are present. After T4 treatment, only $\alpha-\mathrm{Mg}$ diffraction peaks could be observed, and those of $(\mathrm{Mg}$, $\mathrm{Zn})_{3} \mathrm{Gd}$ disappeared for the dissolution, as shown in Fig. 3 b. Because the quantity of the precipitated phase is so small that no new diffraction peaks are visible.
The XRD patterns of T6-treated alloys are similar with those of the T4-treated alloys, so they were not shown in this paper.

In order to understand the compositions of the block precipitated phase after T6 treatment, the enlarged GZ61K-T6 SEM image and EDS spectrum of the block particle are shown in Fig. 4. It shows that the block precipitate is Gd-rich compound, which is probable $\mathrm{Mg}_{5} \mathrm{Gd}$ according to the atomic ratio of $\mathrm{Mg}$ and Gd shown in Fig. 4b. A coarse equilibrium face-centered cubic $\mathrm{Mg}_{5} \mathrm{Gd}$-phase has been precipitated between the grain interiors and the grain boundaries of Mg-Gd binary alloys [28].

\subsection{Mechanical properties}

Figure 5 shows microhardness, yield strength (YS), ultimate tensile strength (UTS), and elongation of the alloys under various conditions. The microhardness increases with the increase of Gd content for the as-cast, T4-treated, and T6-treated alloys. After T4 and T6 treatments, the hardness of the alloy with the same Gd content is improved, as shown in Fig. 5a. The microhardness of the GZ91K-F alloy is $14.3 \%$ higher than that of the GZ31K-F, that of the T4-treated GZ31K, GZ61K, and GZ91K alloys is improved by $17.1,17.4$, and $9.3 \%$ as compared to that of the ascast alloys, and the microhardness of the T6-treated GZ31K, GZ61K, and GZ91K alloys is hardened by $33.9,36.5$, and $25.8 \%$ as compared to that of the as-cast alloys.

The tensile mechanical properties are shown in Figs. 5b-d. The changes of YS and UTS of the alloys basically agree with that of the microhardness. In the same state, the strength is improved with the increase of Gd content. The YS shows 22.5, 26.9, and $31.7 \%$ increasement as the Gd addition increases from 3 to $9 \%$ for the as-cast, T4-treated, and T6-treated alloys, respectively. For the same alloy under different states, the strength after T4 treatment is a little higher than that of the as-cast alloy, and the strength of the T6 alloy is the highest. The YS of the T6-treated GZ31K, GZ61K, and GZ91K alloys is enhanced by 13.5, 17.8, and $22.0 \%$, respectively, as compared to those under as-cast condition, indicating that $\mathrm{T} 6$ treatment is beneficial for strengthening the alloys.

The elongation of the as-cast GZ31K, GZ61K, and GZ91K alloys is $14.3,13.2$, and $12.0 \%$, respectively, showing slight reduction with increasing Gd content. T4 and T6 treatments play complex role in elongation of the alloys. For the GZ31K alloy, the elongation slightly decreases after T4 and T6 treatments. For the GZ61K alloy, the elongation presents no change after $\mathrm{T} 4$ treatment, but it is $72.7 \%$ higher after T6 treatment as compared to that of the as-cast. The elongation of the GZ91K alloys shows increase after T4 and T6 treatments. 

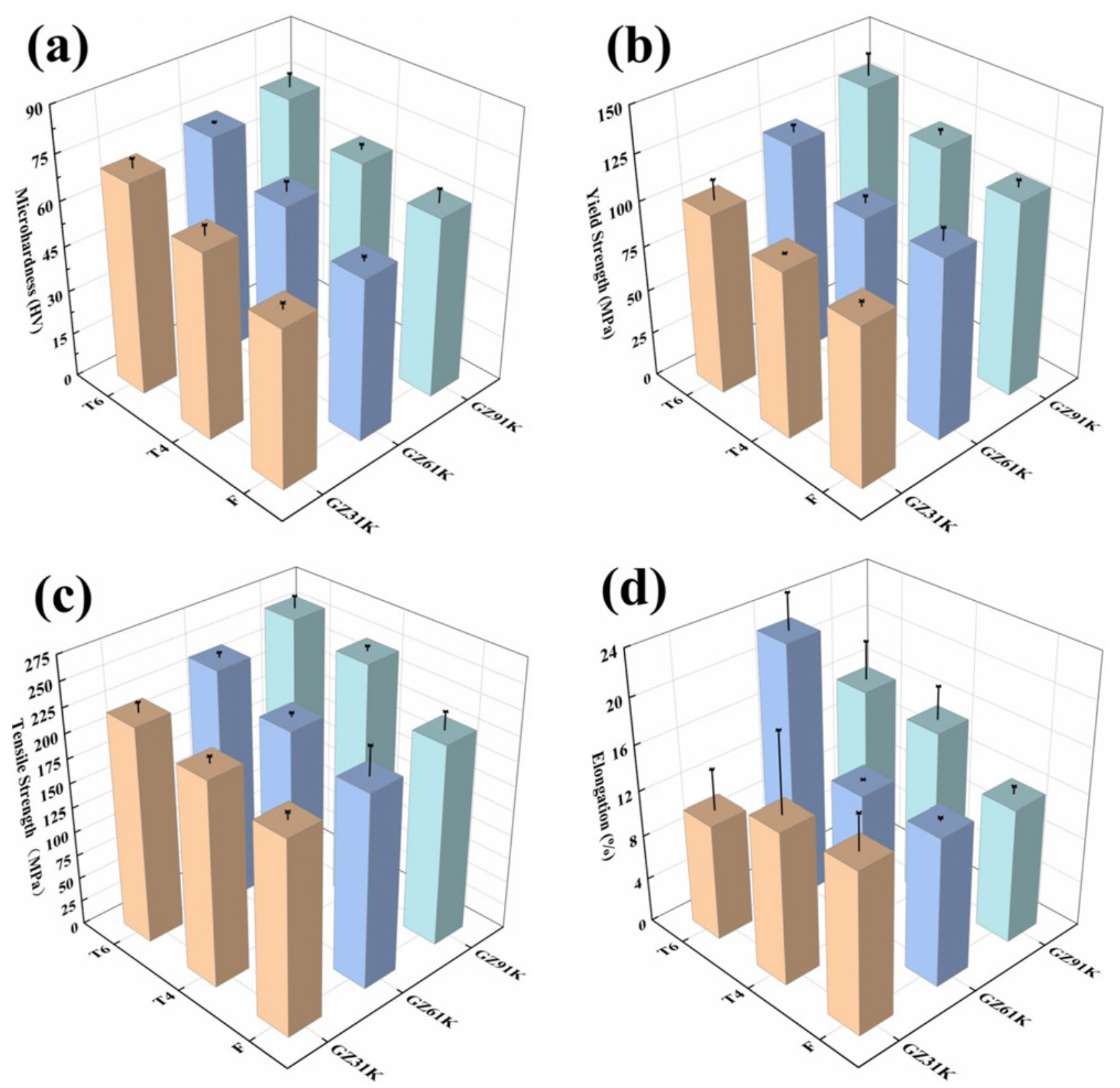

Fig. 5. Mechanical properties of the alloys at different conditions: (a) microhardness, (b) yield strength, (c) ultimate tensile strength, and (d) elongation.

According to the above results of mechanical properties, it can be found that the GZ91K-T6 has the highest hardness $(75.9 \mathrm{HV})$, YS (133 MPa), UTS (261 $\mathrm{MPa})$, and moderate elongation $(15.9 \%)$, while the GZ61K-T6 exhibits the highest elongation (22.8\%). In order to further explore the strengthening mechanisms, the GZ91K alloy was selected to study the nano hardness of different phases, as shown in Fig. 6. The indentation depths of the GZ91K-F matrix and $\beta$-phase are 2950 and $980 \mathrm{~nm}$, indicating the nano hardness of 0.12 and $1.20 \mathrm{GPa}$, and those of the T6 are 2180 and $1100 \mathrm{~nm}$, suggesting the nano hardness of 0.81 and $0.97 \mathrm{GPa}$, respectively.
With the increase of $\mathrm{Gd}$ content, the grain size in the as-cast alloys reduces, and the volume fraction of the $\beta$-phase increases which exhibits higher hardness than the matrix, therefore, the improved strength caused by increasing Gd is mainly attributed to grain refinement and more $\beta$-phase. After T4 treatment, the $\beta$-phase at the grain boundaries is dissolved into the matrix, resulting in solid solution strengthening which is confirmed by nano hardness. In addition, tiny needle-like precipitate is formed which has the effect of precipitation strengthening. Nevertheless, the SFs in the GZ31K and the LPSO structures in the GZ61K and GZ91K alloys disappear, which de- 
teriorates strength of the alloys. Consequently, the strength of the alloys with the same composition only presents slight enhancement after T4 treatment owing to the comprehensively positive effects of solid solution strengthening, precipitation strengthening, and the negative influence of the disappeared SFs or LPSO structures. Compared with T4-treated alloys, the volume fraction of the precipitated phase in the T6-treated alloys is higher, and they distribute more homogeneously, resulting in further improvement of strength.

Nevertheless, the improvement of strength after T4 and T6 treatments is not significant that may be explained by the following reasons. On the one hand, it has been reported that SFs and LPSO structures can significantly improve the mechanical properties of magnesium alloy, because they can accumulate dislocations and prevent their movement $[29,30]$, so the disappeared LPSO structures after T4 and T6 treatments weaken the alloys; on the other hand, the maximum solubility of $\mathrm{Gd}$ in $\mathrm{Mg}$ is $23.49 \mathrm{wt} . \%$ [28], and relatively low addition of Gd cannot cause apparent enhancement of the Mg-Gd series alloys by solid solu-
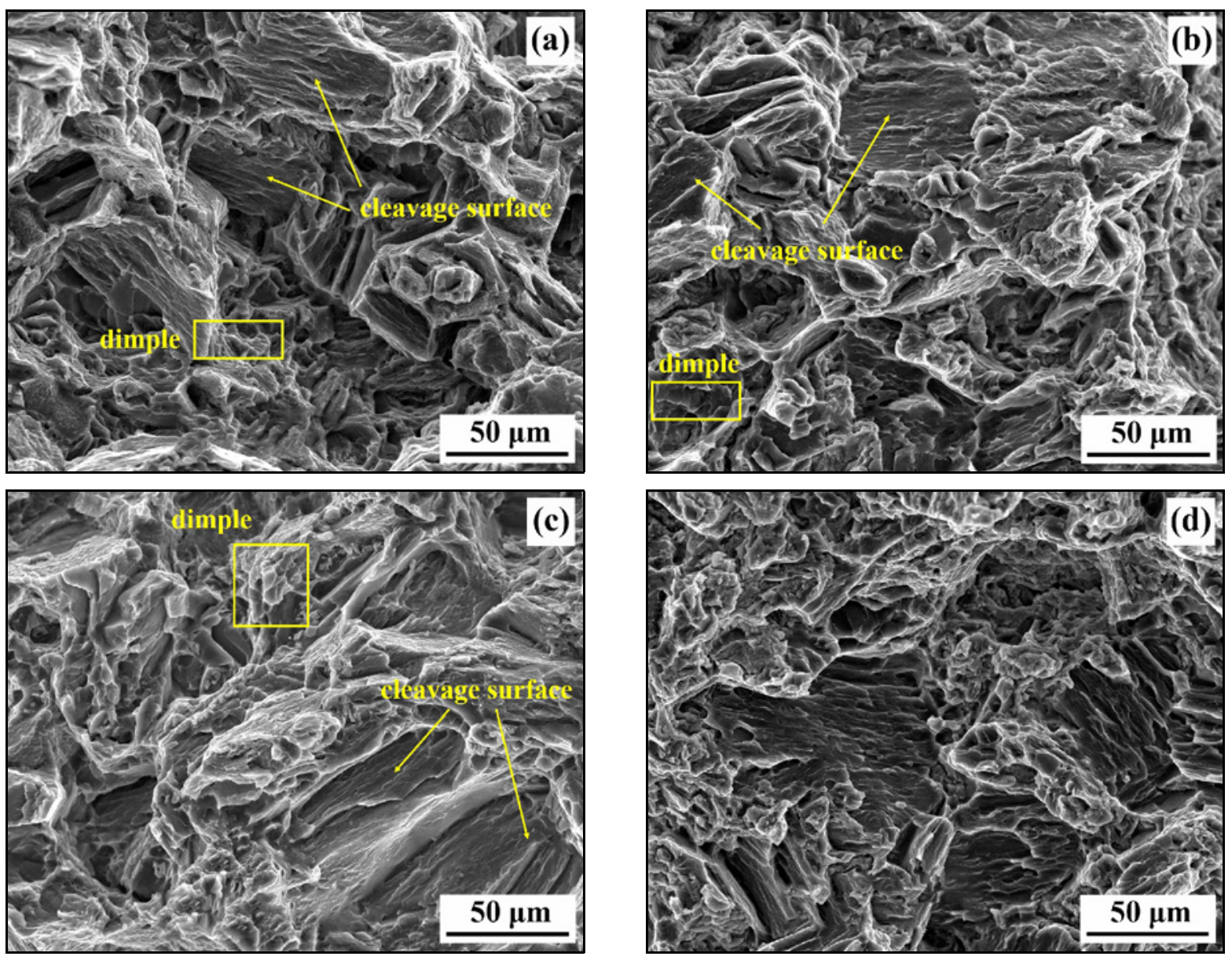

Fig. 7a-d. Fracture morphologies of the Mg- $x$ Gd-1Zn-0.4Zr alloys under different conditions: (a) GZ31K-F, (b) GZ61K-F, (c) GZ91K-F, (d) GZ31K-T4. 

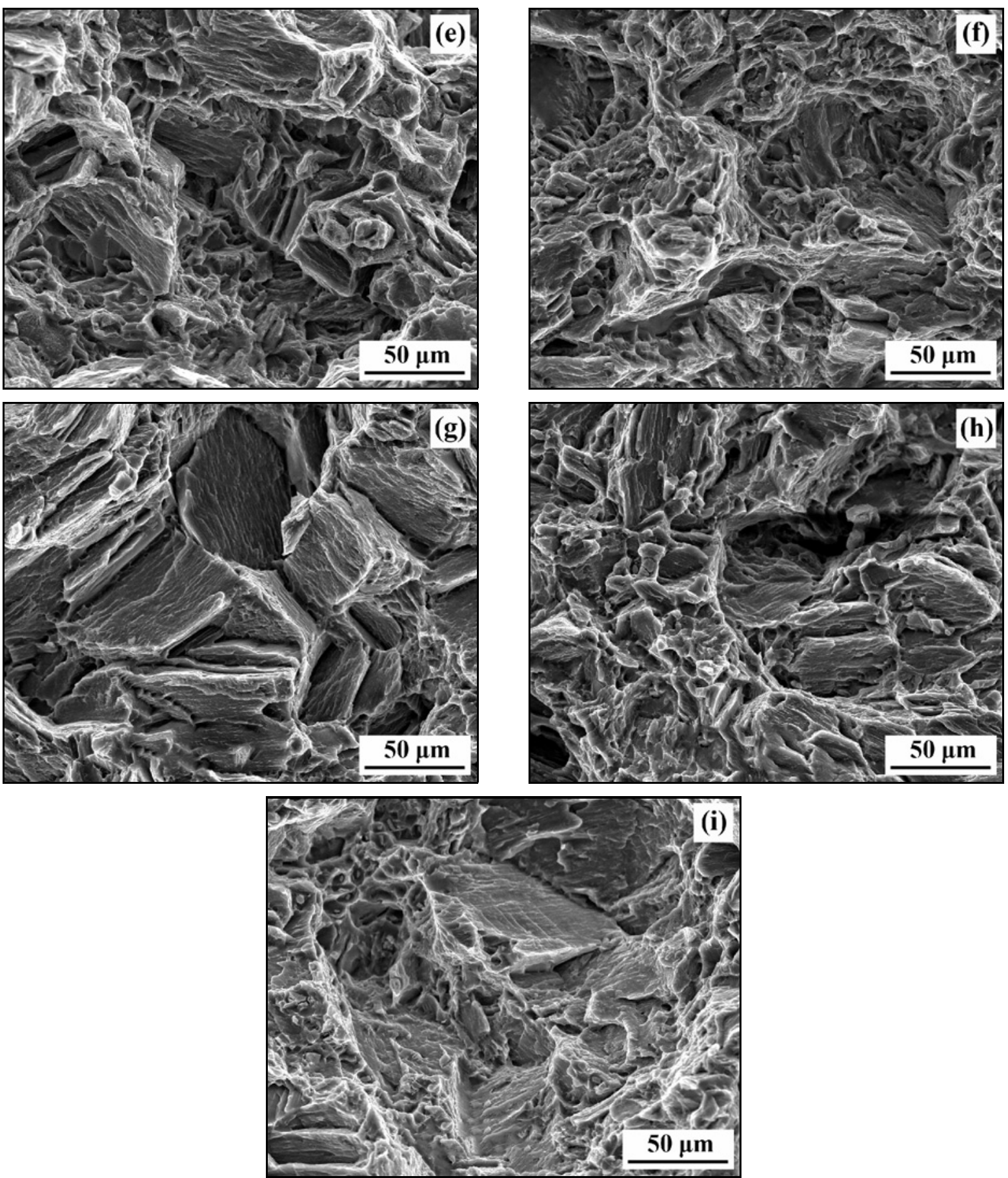

Fig. 7e-i. Fracture morphologies of the Mg- $x$ Gd-1Zn-0.4Zr alloys under different conditions: (e) GZ61K-T4, (f) GZ91K-T4, (g) GZ31K-T6, (h) GZ61K-T6, and (i) GZ91K-T6.

alloys in different states. The fracture consists of dimples and cleavage morphologies. The dimples are the results caused by intergranular fracture for the fine equiaxed grains, and the cleavage surfaces are generated by the fracture of large grains. The increase of $\mathrm{Gd}$ content leads to the increase of the $\beta$-phase which is good for strength and hardness, however, dislocations tend to aggregate at the phase interfaces during tensile deformation, which is prone to the initiation and propagation of cracks [31], and thus, the elongation decreases with increasing $\mathrm{Gd}$ addition even though the microstructure is refined.

For the GZ31K alloy, its elongation decreases after T4 and T6 treatment. It can be seen from Figs. 7a,d,g that the dimples decrease, and the cleavage surfaces increase. Precipitation phase is formed after T4 and T6 treatment, more precipitated particles mean more phase boundaries, which easily cause stress concentra- 
tion, crack initiation and expansion, resulting in lower elongation. For the GZ61K and GZ91K alloys, even though the needle-like precipitates are formed which is harmful to elongation, they are much finer and more homogeneous than the $\beta$-phase. Furthermore, compared with the GZ31K alloy, more volume fraction $\beta$-phase is vanished after heat treatment which is beneficial to elongation. The improved elongation after T4 and T6 treatments for the GZ61K and GZ91K alloys is mainly dominated by the positive effect of the vanished $\beta$-phase but not the negative effect of the increased precipitates, leading to the elongation improvement. Compared with other fracture morphologies, Fig. 7h has more and deeper dimples and the GZ61K-T6 alloy exhibits the highest elongation, this may be attributed to the more homogeneously distributed precipitates as compared to the GZ61K-T4 alloy and less precipitates as compared to the GZ91K-T6 alloy, as shown in Figs. 2e,h,i.

\section{Conclusions}

1. Microstructure of the as-cast $\mathrm{Mg}-x \mathrm{Gd}-1 \mathrm{Zn}-0.4 \mathrm{Zr}$ alloys is composed of $\alpha$-Mg matrix, $\beta$ - $(\mathrm{Mg}, \mathrm{Zn})_{3} \mathrm{Gd}$ at grain boundaries, and lamellar structure at the outer edge of matrix grains. With the increase of Gd content, the volume fraction of $\beta$-phase increases. The $\beta$-phase almost dissolves into matrix and $\mathrm{Zr}$-rich needle-like precipitates are formed after T4 treatment. Except for the increase of precipitates, block $\mathrm{Mg}_{5} \mathrm{Gd}$-phase is formed in the GZ61K and GZ91K alloy after T6 treatment.

2. The microhardness and strength increase with the increase of Gd content mainly due to grain refinement and more strengthened $\beta$-phase, but the elongation decreases slightly for the as-cast alloys.

3. Both T4 and T6 treatments are good for strengthening the alloys owing to solid solution strengthening and precipitation strengthening. The heat treatment responses in GZ91K alloys are remarkable. Yield strength increases from the initial values of $105 \mathrm{MPa}$ in as-cast alloys to the peak hardness value of $121 \mathrm{MPa}$ (after T4) and $133 \mathrm{MPa}$ (after T6). And after T6 treatment, the hardness of the alloy is also increased by $11 \%$.

4. The elongation of the GZ31K alloy decreases but that of the GZ61K and GZ91K alloys improves after T4 and T6 treatments. The elongation of the GZ61K alloy increases from the initial value of $12 \%$ under as-cast condition to the value of $22 \%$ under T6-treated condition.

\section{Acknowledgements}

This project was supported by the Natural Science Foundation of Higher Education Institutions of Jiangsu
Province - Key Project (18KJA430008) and the Practical Innovative Project for Undergraduates of Jiangsu Province (201911276032Y).

\section{References}

[1] Y. F. Zheng, X. N. Gu, F. Witte, Biodegradable metals, Mater. Sci. Eng. R 77 (2014) 1-34. doi:10.1016/j.mser.2014.01.001

[2] W. J. Ding, Opportunities and challenges for the biodegradable magnesium alloys as next-generation biomaterials, Regen. Biomater. 3 (2016) 79-86. doi:10.1093/rb/rbw003

[3] M. Cavojský, V. Trembošová, N. Beronská, Š. Nagy, M. Nosko, Microstructure and mechanical properties of extruded profiles made from pure magnesium powders, Kovove Mater. 57 (2019) 371-376 doi:10.4149/km_2019_6_371

[4] J. Walker, S. Shadanbaz, T. B. F. Woodfield, M. P. Staiger, G. J. Dias, The in vitro and in vivo evaluation of the biocompatibility of $\mathrm{Mg}$ alloys, Biomed Mater. 9 (2014) 015006. doi:10.1088/1748-6041/9/1/015006

[5] A. Lafont, Y. Yang, Magnesium stent scaffolds: DREAMS become reality, Lancet. 387 (2016) 3-4. doi:0.1016/S0140-6736(15)00804-1

[6] X. B. Zhang, J. W. Dai, R. F. Zhang, Z. X. Ba, N. Birbilis, Corrosion behavior of Mg-3Gd-1Zn-0.4Zr alloy with and without stacking faults, J. Magnes. Alloys 7 (2019) 240-248. http://creativecommons.org/licenses/by-nc-nd/4.0/

[7] H. R. Bakhsheshi-Rad, E. Hamzah, M. Medraj, M. H. Idris, A. F. Lotfabadi, M. Daroonparvar, M. A. M. Yajid, Effect of heat treatment on the microstructure and corrosion behaviour of $\mathrm{Mg}-\mathrm{Zn}$ alloys, Mater. Corros. 65 (2014) 999-1006. doi:10.1002/maco.201307492

[8] K. Chen, J. Dai, X. B. Zhang, Improvement of corrosion resistance of magnesium alloys for biomedical applications, Corros. Rev. 33 (2015) 3-4. doi:10.1515/corrrev-2015-0007

[9] X. B. Zhang, J. W. Dai, Q. S. Dong, Z. X. Ba, Y. $\mathrm{J}$. Wu, Corrosion behavior and mechanical degradation of as-extruded $\mathrm{Mg}-\mathrm{Gd}-\mathrm{Zn}-\mathrm{Zr}$ alloys for orthopedic application, J. Biomed. Mater. Res. 108B (2020) 698-708. doi:10.1002/jbm.b.34424

[10] Y. Z. Ma, C. L. Yang, Y. J. Liu, F. S. Yuan, S. S. Liang, H. X. Li, J. S. Zhang, Microstructure, mechanical, and corrosion properties of extruded low-alloyed $\mathrm{Mg}-x \mathrm{Zn}$ -0.2Ca alloys, Int. J. Min. Met. Mater. 26 (2019) 12741284. doi:10.1007/S12613-019-1860-3

[11] A. Mohamed, A. M. El-Aziz, H. G. Breitinger, Study of the degradation behavior and the biocompatibility of $\mathrm{Mg}-0.8 \mathrm{Ca}$ alloy for orthopedic implant applications, J. Magnes. Alloys. 7 (2019) 249-257. doi:10.1016/j.jma.2019.02.007

[12] Y. X. Wang, D. Tie, R. G. Guan, N. Wang, Y. Q. Shang, T. Cui, J. Q. Li, Microstructure, mechanical properties, and degradation behaviors of heat-treated Mg-Sr alloys as potential biodegradable implant materials, J. Mech. Behav. Biomed. 77 (2018) 47-57. doi:10.1016/j.jmbbm.2017.08.028

[13] Z. Li, M. F. Chen, W. Li, H. R. Zheng, C. You, D. B. Liu, F. Jing, The synergistic effect of trace $\mathrm{Sr}$ and $\mathrm{Zr}$ 
on the microstructure and properties of a biodegradable Mg-Zn-Zr-Sr alloy, J. Alloys Compd. 702 (2017) 290-302. doi:10.1016/j.jallcom.2017.01.178

[14] S. Dai, F. Wang, D. Z. Ma, Z. Wang, Z. Liu, P. L. Mao, Enhanced strengthening by two-step progressive solution and aging treatment in AM50-4\%(Zn, Y) magnesium alloy, Trans. Nonferr. Metal. Soc. China 28 (2018) 2419-2426. doi:10.1016/S1003-6326(18)64888-5

[15] Q. Y. Huang, Y. Liu, A. Y. Zhang, H. X. Jiang, H. C. Pan, X. H. Feng, C. L. Yang, T. J. Luo, Y. J. Li, Y. S. Yang, Age hardening responses of as-extruded $\mathrm{Mg}-2.5 \mathrm{Sn}-1.5 \mathrm{Ca}$ alloys with a wide range of $\mathrm{Al}$ concentration, J. Mater. Sci. Technol. 38 (2020) 39-46. doi:10.1016/j.jmst.2019.06.025

[16] Z. B. Ding, R. P. Lu, H. Hou, Effect of heat treatment on microstructure and mechanical properties of alloy Mg- $10 \%$ Gd- $3 \% \mathrm{Y}-0.6 \% \mathrm{Zr}$, Met. Sci. Heat Treat. 61 (2019) 434-439. doi:10.1007/s11041-019-00441-y

[17] J. S. Wang, X. B. Li, Simultaneously improving strength and ductility of AZ91-type alloys with minor Gd addition, J. Alloys Compd. 803 (2019) 689-699. doi:10.1016/i.jallcom.2019.06.313

[18] M. B. Yang, M. D. Hou, J. Zhang, F. S. Pan, Effects of $\mathrm{Ce}, \mathrm{Y}$ and $\mathrm{Gd}$ additions on as-cast microstructure and mechanical properties of $\mathrm{Mg}$-3Sn-2Sr magnesium alloy, Trans. Nonferr. Metal. Soc. China 24 (2014) 24972506. doi:10.1016/i.msea.2009.06.040

[19] X. B. Zhang, Z. X. Ba, Z. Z. Wang, Y. J. Wu, Y. J. Xue, Effect of LPSO structure on mechanical properties and corrosion behavior of as-extruded GZ51K magnesium alloy, Mater. Lett. 163 (2016) 250-253. doi:10.1016/j.matlet.2015.10.084

[20] X. B. Zhang, Q. Wang, F. B. Chen, Y. J. Wu, Z. Z. Wang, Relation between LPSO structure and biocorrosion behavior of biodegradable GZ51K alloy, Mater. Lett. 138 (2015) 212-215. doi:10.1016/j.matlet.2014.09.133

[21] X. B. Zhang, Z. X. Ba, Q. Wang, Y. J. Wu, Z. Z. Wang, Uniform corrosion behavior of GZ51K alloy with long period stacking ordered structure for biomedical application, Corros. Sci. 88 (2014) 1-5. doi:10.1016/j.corsci.2014.07.004

[22] X. Zhang, S. K. Kairy, J. Dai, N. Birbilis, A closer look at the role of nanometer scale solute-rich stacking faults in the localized corrosion of a magnesium alloy GZ31K, J. Electrochem. Soc. 165 (2018) C310-C316. doi:10.1149/2.0391807jes
[23] X. B. Zhang, Z. X. Ba, Z. Z. Wang, Y. J. Xue, Microstructures and corrosion behavior of biodegradable Mg-6Gd- $x$ Zn- $0.4 \mathrm{Zr}$ alloys with and without long period stacking ordered structure, Corros. Sci. 105 (2016) 68-77. doi:10.1016/j.corsci.2016.01.004

[24] A. Srinivasan, Y. Huang, C. L. Mendis, C. Blawert, K. U. Kainer, N. Hort, Investigations on microstructures, mechanical and corrosion properties of Mg-Gd-Zn alloys, Mater. Sci. Eng. A 595 (2014) 224-234. doi:10.1016/j.msea.2013.12.016

[25] W. Rong, Y. Zhang, Y. Wu, Y. Wang, M. Sun, J. Chen, Y. Wang, J. Y. Han, L. M. Peng, H. X. Ding, Effects of $\mathrm{Zr}$ and $\mathrm{Mn}$ additions on formation of LPSO structure and dynamic recrystallization behavior of $\mathrm{Mg}$ -15Gd-1Zn alloy, J. Alloys Compd. 692 (2016) 805816. doi:10.1016/i.jallcom.2016.09.068

[26] J. Zhang, X. B. Zhang, Q. H. Liu, S. J. Yang, Z. Z. Wang, Effects of load on dry sliding wear behavior of Mg-Gd-Zn-Zr alloys, J. Mater. Sci. Technol. 33 (2017) 645-651. doi:10.1016/j.jmst.2016.11.014

[27] X. B. Zhang, Z. X. Ba, Z. Z. Wang, Y. J. Xue, Q. Wang, Microstructure and biocorrosion behaviors of solution treated and as-extruded $\mathrm{Mg}-2.2 \mathrm{Nd}-x \mathrm{Sr}-0.3 \mathrm{Zr}$ alloys, Trans. Nonferr. Metal. Soc. China 24 (2014) 3797-3803. doi:10.1016/S1003-6326(14)63535-4

[28] N. Hort, Y. Huang, D. Fechner, M. Strömer, C. Blawert, F. Witte, C. Vogt, H. Drücker, R. Willumeit, K. U. Kainer, F. Feyerabend, Magnesium alloys as implant materials - Principles of property design for Mg-RE alloys, Acta Biomater. 6 (2010) 1714-1725. doi:10.1016/j.actbio.2009.09.010

[29] L. Zhang, J. H. Zhang, C. Xu, Y. B. Jing, J. P. Zhuang, R. Z. Wu, M. L. Zhang, Formation of stacking faults for improving the performance of biodegradable Mg-Ho-Zn alloy, Mater. Lett. 133 (2014) 158-162. doi:10.1016/j.matlet.2014.06.171

[30] K. Hagihara, A. Kinoshita, Y. Sugino, M. Yamasaki, Y. Kawamura, H. Y. Yasuda, Y. Umakoshi, Effect of long-period stacking ordered phase on mechanical properties of $\mathrm{Mg}_{97} \mathrm{Y}_{1} \mathrm{Zn}_{2}$ extruded alloy, Acta Mater. 58 (2010) 6282-6293. doi:10.1016/j.actamat.2010.07.050

[31] W. Fu, R. H. Wang, H. Xue, J. Kuang, J. Y. Zhang, G. Liu, J. Sun, Effects of Zr addition on the multi-scale second-phase particles and fracture behavior of $\mathrm{Mg}$ 3Gd-1Zn alloy, J. Alloys Compd. 747 (2018) 197-210. doi:10.1016/j.jallcom.2018.02.328 